the Earth's oceans. The combination of tidal heating and that due to the decay of radioactive elements expected in the rock would have provided temperatures of at least $200{ }^{\circ} \mathrm{C}$; the pressure at the interface between core and mantle can be estimated at a few thousand bars.

This pressure-cooker would have been in operation for a hundred million years or so, during which time the carbon- and nitrogen-bearing molecules dissolved in the water could have undergone very extensive chemical reworking. Stevenson and $\mathrm{Gandhi}^{9}$ proposed that $\mathrm{CO}$ might be chemically destroyed in such a system. Shock and McKinnon have undertaken quantitative examination of the products of this chemistry.

Their model considers 18 compounds of the elements $\mathrm{C}, \mathrm{N}, \mathrm{O}$ and $\mathrm{H}$, and calculates their equilibrium abundances under the assumption that Triton's hydrothermal system is buffered by particular assemblages of rocks, which determine the redox state of the chemical system. The most striking of their results is that, for a very wide range of redox states of the liquid water (corresponding to a range of possible mineral assemblages), $\mathrm{CO}$ is a minor species relative to $\mathrm{CO}_{2}$. That is, cooking a mix of carbon-bearing species in aqueous solution at high temperature and pressure will substantially decrease the carbon monoxide abundance.

The model further predicts that $\mathrm{N}_{2}$ is more abundant than $\mathrm{CO}$ over a wide range of plausible redox states. For progressively more reducing mineral assemblages, $\mathrm{N}_{2}$ does give way to $\mathrm{NH}_{3}$ as the predominant nitrogen-bearing species, and eventually no longer dominates over CO. However, the range over which the $\mathrm{N}_{2}$ dominates is large, so chemical alteration in a hydrothermal system appears capable of producing the carbon and nitrogen inventories seen on Triton's surface.

A number of questions are raised by the work. The abundance of $\mathrm{CH}_{4}$ is a particularly problematic one, as that molecule is seen on the surfaces of both Triton and Pluto. Shock and McKinnon exclude methane from the chemical system, arguing that in terrestrial hydrothermal systems it does not readily equilibrate with the other compounds except at rather high temperatures. In effect, $\mathrm{CH}_{4}$ and other light hydrocarbons are kinetically inhibited from participating in the chemistry. This may well be the case in terrestrial hydrothermal systems, but the relevant dynamical time constants in Triton's system could be different.

A second issue is how these materials find their way to the surface, and the timing of surface extrusion relative to hydrothermal cycling. The carbon- and nitrogen-bearing ices must be extruded after most of the mantle material has been hydrothermally altered, which sets constraints on the timing of outgassing, volcanic extrusion, and mantle cooling and refreezing. These are not addressed in detail in the paper, but will be grist to the mill of planetologists who model such processes.

Pluto's composition represents a third question raised by this model. The inventory of $\mathrm{CH}_{4}$ and $\mathrm{CO}$ on Pluto is modestly larger than on Triton, and from the lack of detection of $\mathrm{CO}_{2}$ one can estimate an upper limit which is roughly a third of that on Triton. Thus one might argue, and the authors do, that Pluto has been subjected to less hydrothermal processing than has Triton. Not much less, though, because $\mathrm{CO}$ remains a very minor component relative to $\mathrm{N}_{2}$. So if hydrothermal processing has determined the surface inventory on both bodies, Pluto too must once have had a liquid water mantle. This sets constraints on Pluto's thermal history, arguing that in spite of the absence of tidal dissipation Pluto must have undergone substantial heating. Perhaps we must face the fact that neither object represents an unaltered sample of the primitive material of the outer Solar System.

As do all good models, Shock and McKinnon's story leaves us with an intriguing punchline. Hydrothermal alteration acts not just to destroy $\mathrm{CO}$ but also to produce a suite of organic compounds of biological interest, including methanol, formic acid, urea and glycine, with abundances dependent on the buffering mineral assemblages.

The lack of spectroscopic detection of such species on Triton and Pluto may reflect the redox state of the internal chemistry, their failure to be erupted on the surface, burial by more volatile ices, or just the difficulties associated with spectroscopic examination of distant, dim bodies. Nonetheless, their presence in the theoretical soup invoked for Triton's interior reinforces the fact that many of the raw materials for life can be made in many places in the cosmos. Hydrothermal alteration itself probably occurred in the parent bodies of asteroids and hence meteorites, producing organic compounds which found their way to the Earth in the past, and continue to do so.

Jonathan 1. Lunine is at the Lunar and Planetary Laboratory, University of Arizona, Tucson, Arizona 85721, USA.

\footnotetext{
Shock, E. L. \& McKinnon, W. B. Icarus 106, 464-477 (1993).

. Van Dishoeck, E. F et al in Protostars and Planets III (eds Levy, E. H. \& Lunine, J. I) 163-241 (University of Arizona Press, Tucson, 1993)

3. Mumma, M. J. et al. in Protostars and Planets III (eds Levy. E. H. \& Lunine, J. I.) 1177-1252 (University of Arizona Press, Tucson, 1993)

4. Stern. S.A. Icarus 90, 271-281(1991)

. Goldreich, P. etal Science 245, 500-504 (1989)

Cruikshank. D. P. et al. Science 261, 742-745 (1993) Owen. T. C. et al. Science 261, 745-748 (1993)

8. Lewis, J. S. \& Prinn, R. G. Astrophys, J. 238, 357-364 (1980).

9. Stevenson, D. J.\& Gandhi, A. S. Lunar planet. Sci. XXI
} 1202-1203(1990)

\section{Groaning boards}

THE traditional coal mine used wooden roof supports. If a roof fall threatened, they began to creak: a useful early warning. Wood creaks under load because individual fibres start to slip jerkily past one another. Similarly, metallic tin 'cries' when bent. One small volume of the lattice dislocates under stress; this increases the load on its neighbours and they yield in turn. So dislocations are triggered in sudden cascades. Daedalus reckons that every material must creak out its own overload warning in this way. Sadly, such warnings are usually too high to hear.

Daedalus is now listening to them. He is sticking piezoelectric contact microphones onto bars of test material, and then bending or stretching them in various ways. The microphone output goes to a frequency shifter, of the type used by biologists to listen to the ultrasonic cries of bats. Once converted to audio, the creaks and groans of each specimen should reveal its state of stress, and how close it is to failing.

With luck, a specimen will emit a characteristic cry for each type of applied stress. When that stress is relaxed, internal hysteresis will return the lattice to its original shape by a slightly different route, and it will emit a different return cry. Once Daedalus has cracked the code, any engineering structure could be fitted with microphones. It would thereafter provide a running commentary on its changing conditions of stress.

Aerospace engineers will be the first beneficiaries. A few microphones, each picking up the cries of a wide volume of structure, should give total surveillance of even a big aircraft. The system will be calibrated by known loads; thereafter computerized signal analysis will easily locate and disentangle the simultaneous chatter of many different spars and panels. Usually the groans of relaxation will alternate reassuringly with the creaks of stress. The shriek of brief overload, the crackle of plastic flow, the insidious muttering of advancing creep and fatigue, will echo in from the most distant and inaccessible points. A microphone on a turbine shaft might even detect creep murmurings transmitted from the blades themselves.

Engineers will rush to fit Daedalus's system to structures old and new. A few microphones will monitor them more comprehensively than the densest web of strain gauges. In countries such as Britain, it will allow elderly cranes, Victorian bridges and crumbling infrastructure generally to be patched up and kept going exactly to the point of collapse. David Jones 